\title{
Lycopene attenuates high glucose-mediated apoptosis in MPC5 podocytes by promoting autophagy via the PI3K/AKT signaling pathway
}

\author{
QINGFEN WANG ${ }^{1}$, RUI LI $^{1}$, ZHI XIAO $^{1}$ and $\mathrm{CUN} \mathrm{HOU}^{2}$ \\ ${ }^{1}$ Department of Nephrology, Binzhou People's Hospital, Binzhou, Shandong 255610; \\ ${ }^{2}$ Department of Nephrology, Jining No. 1 People's Hospital, Jining, Shandong 272011, P.R. China
}

Received August 27, 2019; Accepted May 29, 2020

DOI: $10.3892 /$ etm.2020.8999

\begin{abstract}
Podocyte injury serves an important role during the progression of diabetic nephropathy (DN), and lycopene (Lyc) may display a potential protective effect against DN progression. The effects of Lyc on high glucose (HG)-induced podocyte apoptosis and the underlying mechanisms are not completely understood; therefore, the present study aimed to investigate the effects of Lyc on HG-induced MPC5 podocyte apoptosis and the underlying mechanism. In the present study, MPC5 podocytes were exposed to $\mathrm{HG}$ and different doses of Lyc. MPC5 podocyte viability and apoptosis were assessed by performing the MTT assay and flow cytometry, respectively. To explore the effects of Lyc on the PI3K/AKT signaling pathway and autophagy, LY294002 (LY) and 3-methyladenine (3-MA) were used as PI3K and autophagy inhibitors, respectively. The expression levels of nephrin, podocin, apoptosis-related proteins (Bax, Bcl-2 and cleaved caspase-3), autophagy-related proteins [Beclin-1 and microtubule associated protein 1 light chain 3 (LC3)II/LC3I] and certain key proteins involved in the PI3K/AKT signaling pathway were measured via western blotting. The results suggested that Lyc reversed the inhibitory effect of $\mathrm{HG}$ on cell viability, and the protein expression levels of nephrin and podocin, as well as the promoting effect of $\mathrm{HG}$ on MPC5 podocyte apoptosis. In addition, under HG conditions, Lyc upregulated the phosphorylation levels of PI3K and AKT, and reduced HG- and LY-mediated MPC5 podocyte apoptosis. Moreover, Lyc further increased HG-induced protein expression levels of Beclin-1 and LC3II/LC3I, and attenuated LY-mediated inhibition of HG-induced MPC5 podocyte autophagy. In addition, the effects of Lyc on HG-mediated MPC5 podocyte apoptosis were alleviated by
\end{abstract}

Correspondence to: Dr Cun Hou, Department of Nephrology, Jining No. 1 People's Hospital, 6 Jiankang Road, Rencheng, Jining, Shandong 272011, P.R. China

E-mail: houcun_chou@163.com

Key words: MPC5 podocytes, apoptosis, autophagy, lycopene, $\mathrm{PI} 3 \mathrm{~K} / \mathrm{AKT}$ signaling pathway
3-MA. Therefore, the present study suggested that Lyc may protect against HG-induced MPC5 podocyte apoptosis by promoting autophagy activity via activation of the PI3K/AKT signaling pathway.

\section{Introduction}

Long-term hyperglycemia, which causes diabetic nephropathy (DN), is a frequent and principal cause of death and morbidity among patients with diabetes (1). DN is one of the most common diabetic complications that often occurs in patients with type 1 and 2 diabetes (2), and is also major risk factor for chronic kidney diseases (3). Moreover, patients with DN are highly likely to develop end-stage renal disease, which ultimately requires renal replacement (4); therefore, the current treatments, such as intensive blood pressure regulation focused on blockage of the renin-angiotensin system, and the regulation of hyperglycaemia, dyslipidaemia and albuminuria, are not effective (5).

Previous studies have demonstrated that factors, such as mesangial extracellular matrix deposition, thickening of the basement membrane, glomerular pericytes, and decreased numbers of podocytes and microvascular alterations, are closely related to renal dysfunction in DN (6-9). Terminally differentiated podocytes are specialized glomerular visceral endothelial cells that exert critical effects on the formation of the glomerular filtration barrier and inhibition of urinary protein loss (10). Damages to the glomerular filtration barrier increase the glomerular filtration rate and albuminuria (11). Podocytes damage contribute to proteinuria and the development of glomerulosclerosis (11). Collectively, the aforementioned findings suggested that protecting against podocyte injury may serve as a potential therapeutic strategy for DN progression. In addition, podocyte injury includes loss of podocytes in the glomerulus (12), which is attributed to hyperglycemia-induced apoptosis, and directly leads to proteinuria and glomerular sclerosis (13). Therefore, the potential therapeutic application of mitigating podocyte apoptosis for the progression of DN requires further investigation.

Lycopene (Lyc), a nutraceutical, is a natural pigment that is rich in tomatoes and other plants (14). Lyc not only displays remarkable active oxygen scavenging abilities and 
antioxidation activities against quenching singlet oxygen and lipid peroxidation, but also displays anticancer, anti-inflammatory and antiapoptotic effects (15-17). Moreover, Li et al (18) demonstrated that Lyc can improve DN progression in diabetic model rats, and $\mathrm{Ni}$ et al (19) reported that Lyc can enhance autophagy and attenuate apoptosis to protect against high glucose (HG)-induced podocyte injury.

Although the effects of Lyc on podocyte injury and apoptosis have attracted increasing attention, the exact mechanism underlying how Lyc exerts its protective effect on HG-induced podocyte apoptosis is not completely understood. Therefore, the present study explored the protective effect of Lyc on HG-induced MPC5 podocyte apoptosis and the underlying mechanism.

\section{Materials and method}

Cell culture. Conditionally immortalized mouse podocytes (MPC5) were purchased from American Tissue Culture Collection. MPC5 podocytes were cultured and induced for cell proliferation and differentiation as previously described (20). MPC5 podocytes were cultured in RPMI 1640 medium (Beijing Solarbio Science \& Technology Co., Ltd.) containing 10\% FBS (Sigma-Aldrich; Merck KGaA) and $10 \mathrm{U} / \mathrm{ml}$ recombinant mouse interferon- $\gamma$ (IFN $\gamma$; Peprotech, Inc.) at $33^{\circ} \mathrm{C}$ with $5 \% \mathrm{CO}_{2}$ and $95 \%$ relative humidity.

To stimulate cell differentiation, MPC5 podocytes were subcultured in RPMI-1640 containing 10\% FBS without mouse IFN $\gamma$ for $10-14$ days at $37^{\circ} \mathrm{C}$ with $5 \% \mathrm{CO}_{2}$ to reach $80-90 \%$ confluence. Prepared MPC5 podocytes were used for subsequent experiments.

Cell viability assay. The viability of differentiated MPC5 podocytes was determined using an MTT assay (Sigma-Aldrich; Merck KGaA), according to the manufacturer's protocol. MPC5 podocytes were seeded $\left(1 \times 10^{4}\right.$ cells/well) into 96-well plates and incubated with RPMI-1640 supplemented with $10 \%$ FBS for $24 \mathrm{~h}$ at $37^{\circ} \mathrm{C}$. As previously described (21), MPC5 podocytes were divided into seven groups: i) Normal group (NG; $5.5 \mathrm{mM}$ glucose); ii) hypertonic group [HP; $5.5 \mathrm{mM}$ glucose and $19.5 \mathrm{mM}$ mannitol (Sigma-Aldrich; Merck $\mathrm{KGaA})$; iii) HG (25 mM glucose); iv) HG and low-concentration Lyc treatment group [HG + L-Lyc; $25 \mathrm{mM}$ glucose + $3.125 \mathrm{mM}$ Lyc (Sigma-Aldrich; Merck KGaA)]; v) HG and high-concentration Lyc treatment group (HG + H-Lyc; $25 \mathrm{mM}$ glucose + $12.5 \mathrm{mM}$ Lyc); vi) low-concentration Lyc treatment group (L-Lyc; $3.125 \mathrm{mM}$ Lyc); and vii) high-concentration Lyc treatment group (H-Lyc; $12.5 \mathrm{mM}$ Lyc). All groups were treated at $37^{\circ} \mathrm{C}$ for $48 \mathrm{~h}$. Subsequently, $20 \mu \mathrm{l}$ MTT solution $(5 \mathrm{mM})$ was added to each well and incubated for another $4 \mathrm{~h}$ at $37^{\circ} \mathrm{C}$. The absorbance of each well was measured at a wavelength of $570 \mathrm{~nm}$ using a microplate reader. The cell viability in individual groups of cells was calculated as the optical density (OD) value of experiments/the OD values of control cells.

Western blotting. MPC5 podocyte protein expression was assessed via western blotting as previously described (11). Briefly, MPC5 podocytes were washed twice with PBS. Subsequently, total protein was extracted from MPC5 podocytes using RIPA buffer (Thermo Fisher Scientific, Inc.) with a complete protease inhibitor cocktail (Roche Diagnostics $\mathrm{GmbH}$ ) on ice for $30 \mathrm{~min}$. Subsequently, the supernatants were collected by centrifugation at $12,000 \mathrm{x}$ g for $10 \mathrm{~min}$ at $4^{\circ} \mathrm{C}$ and total protein was quantified using the BCA protein assay kit (Beijing Solarbio Science \& Technology Co., Ltd.).

Subsequently, protein (30 $\mu \mathrm{g} /$ lane) was separated via $12 \%$ SDS-PAGE and transferred onto PVDF membranes (EMD Millipore), which were then blocked with 5\% non-fat milk in TBST (0.1\% Tween-20) for $1 \mathrm{~h}$ at room temperature. The membranes were incubated at $4{ }^{\circ} \mathrm{C}$ overnight with the following primary antibodies: Anti-nephrin (rabbit; 1:400; cat. no. ab58968; Abcam), anti-podocin (rabbit; 1:1,300; cat. no. ab50339; Abcam), anti-Bcl-2 (rabbit; 1:1,000; cat. no. ab59348; Abcam), anti-Bax (rabbit; 1:1,000; cat. no. ab32503; Abcam), anti-cleaved (C) caspase-3 (rabbit; 1:500; cat. no. ab2302; Abcam), anti-phosphorylated (p)-PI3K (rabbit; 1:1,000; cat. no. 4228; Cell Signaling Technology, Inc.), anti-PI3K (rabbit; 1:1,000; cat. no. 4292; Cell Signaling Technology, Inc.), anti-p-AKT (rabbit; 1:1,000; cat. no. 9271; Cell Signaling Technology, Inc.), anti-AKT (rabbit; 1:1,000; cat. no. 9272; Cell Signaling Technology, Inc.), anti-Beclin-1 (rabbit; 1:200; cat. no. ab62557; Abcam), anti-microtubule associated protein 1 light chain 3 (LC3)I (rabbit; 1:1,000; cat. no. 12741; Cell Signaling Technology, Inc.), anti-LC3II (rabbit; 1:1,000; cat. no. 12741; Cell Signaling Technology, Inc.) and anti-GAPDH (rabbit; 1:10,000; cat. no. ab181602; Abcam). Following primary incubation, the membranes were incubated with a horseradish peroxidase-conjugated goat anti-rabbit secondary antibody (1:2,000; cat. no. ab205718; Abcam) at room temperature for $2 \mathrm{~h}$. Subsequently, the membranes were washed three times with TBST [50 mM Tris- $\mathrm{HCl}(\mathrm{pH} \mathrm{8.0)}$; $150 \mathrm{mM} \mathrm{NaCl} ; 0.05 \%$ Tween-20] for $10 \mathrm{~min}$. Protein bands were visualized using an ECL detection kit (Promega Corporation). Protein expression levels were quantified using ImageJ software (version 1.8.0; National Institute of Health) with GAPDH as the loading control.

Flow cytometry analysis. MPC5 podocytes $\left(1 \times 10^{5}\right.$ cells/well) were cultured in 24-well plates and FBS-starved for $24 \mathrm{~h}$ at $37^{\circ} \mathrm{C}$. To investigate the effects of Lyc on HG-induced MPC5 podocyte injury, cells were then divided into five groups: i) NG; ii) $\mathrm{HG}$; iii) $\mathrm{HG}+\mathrm{L}-\mathrm{Lyc}$; iv) $\mathrm{HG}+\mathrm{H}-\mathrm{Lyc}$; and v) H-Lyc. Podocytes were treated with the aforementioned treatment for $48 \mathrm{~h}$ at $37^{\circ} \mathrm{C}$. MPC5 podocytes $\left(1 \times 10^{5}\right.$ cells/well $)$ were cultured in 24-well plates and FBS-starved for $24 \mathrm{~h}$ at $37^{\circ} \mathrm{C}$. To investigate the effects of Lyc on HG-induced MPC5 podocyte injury, and the PI3K/AKT pathway, cells were divided into five groups: i) $\mathrm{NG}$; ii) $\mathrm{HG}$; iii) $\mathrm{HG}+\mathrm{H}-\mathrm{Lyc}$; iv) $\mathrm{HG}+\mathrm{LY}$; and v) $\mathrm{HG}+\mathrm{H}-\mathrm{Lyc}+\mathrm{LY}$. Podocytes were treated with the normal glucose, high glucose, high glucose with H-Lyc, high glucose with the PI3K inhibitor $20 \mu \mathrm{M} \mathrm{LY}$ (cat. no. S1105; Selleck Chemicals) or $20 \mu \mathrm{M}$ LY for $48 \mathrm{~h}$ at $37^{\circ} \mathrm{C}$, respectively. MPC5 podocytes $\left(1 \times 10^{5}\right.$ cells/well $)$ were cultured in 24-well plates and FBS-starved for $24 \mathrm{~h}$ at $37^{\circ} \mathrm{C}$. To investigate whether Lyc has an effect on HG-induced MPC5 podocyte injury through autophagy, the cells were divided into five groups: i) $\mathrm{NG}$; ii) $\mathrm{HG}$; iii) $\mathrm{HG}+\mathrm{H}-\mathrm{Lyc}$; iv) $\mathrm{HG}+3-\mathrm{MA}$; and v) $\mathrm{HG}+\mathrm{H}-\mathrm{Lyc}+3-\mathrm{MA}$. MPC5 podocytes in the $\mathrm{HG}+$ 3-MA and $\mathrm{HG}+\mathrm{H}-\mathrm{Lyc}+3-\mathrm{MA}$ groups were pretreated with 

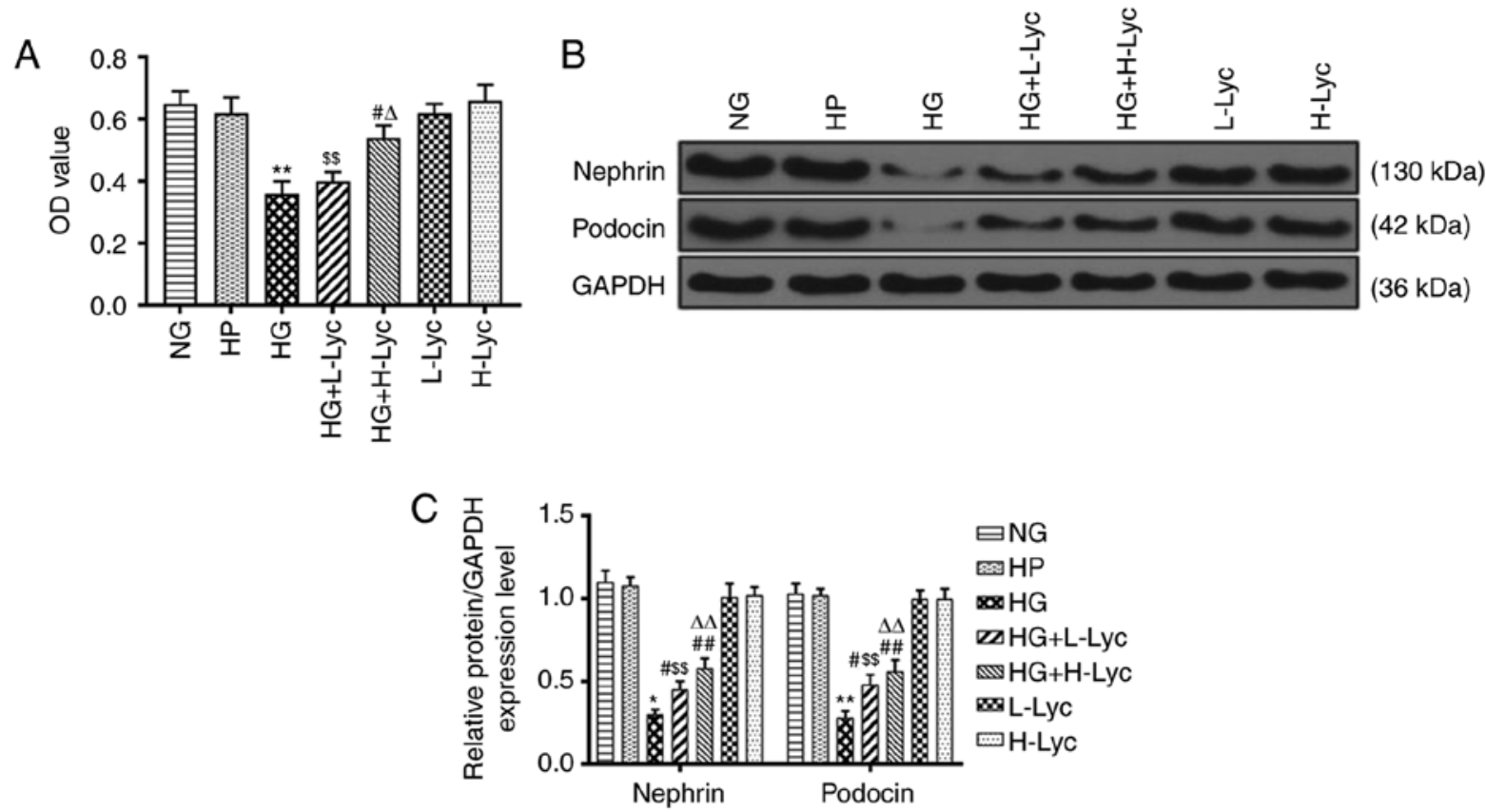

Figure 1. Lyc protects against HG-induced MPC5 podocyte injury. MPC5 podocytes were divided into the following groups: i) NG (5.5 mM glucose); ii) HP (5.5 mM glucose + $19.5 \mathrm{mM}$ mannitol); iii) HG (25 mM glucose); iv) HG + L-Lyc (25 mM glucose + $3.125 \mathrm{mM}$ Lyc); v) HG + H-Lyc (25 mM glucose + $12.5 \mathrm{mM}$ Lyc); vi) L-Lyc (3.125 mM Lyc); and vii) H-Lyc (12.5 mM Lyc). (A) Effect of Lyc on MPC5 podocyte cell viability. Nephrin and podocin protein expression levels were (B) determined by western blotting and (C) semi-quantified. ${ }^{*} \mathrm{P}<0.05$ and ${ }^{* *} \mathrm{P}<0.01 \mathrm{vs}$. NG; ${ }^{\#} \mathrm{P}<0.05$ and ${ }^{\# \#} \mathrm{P}<0.01 \mathrm{vs}$. HG; ${ }^{\$ \$} \mathrm{P}<0.01$ vs. L-Lyc; ${ }^{\circ} \mathrm{P}<0.05$ and ${ }^{\Delta} \mathrm{P}<0.01$ vs. H-Lyc. Lyc, lycopene; NG, normal glucose; HP, hypertonic; HG, high glucose; L-Lyc, low-concentration lycopene; H-Lyc, high-concentration lycopene; OD, optical density.

5 mM 3-MA (cat. no. M9281; Sigma-Aldrich; Merck KGaA), an inhibitor of autophagy initiation, for $2 \mathrm{~h}$ at $37^{\circ} \mathrm{C}$, then treated with high glucose or H-Lyc additively for $48 \mathrm{~h}$ at $37^{\circ} \mathrm{C}$. The cells of NG, HG and $\mathrm{HG}+\mathrm{H}$-Lyc groups were treated with normal glucose, high glucose and high glucose $+\mathrm{H}-\mathrm{Lyc}$ for $48 \mathrm{~h}$ at $37^{\circ} \mathrm{C}$, respectively. Subsequently, treated MPC5 podocytes were collected in PBS and stained using the Annexin V-FITC Apoptosis Detection kit (cat. no. CA1020; Beijing Solarbio Science \& Technology Co., Ltd.). After washing, MPC5 podocyte apoptosis was determined by flow cytometry using the Accuri $^{\mathrm{TM}}$ C6 flow cytometer (BD Biosciences) and Cell Quest software (version 3.3; BD Biosciences). The apoptotic rate was calculated as follows: Apoptotic rate=early + late apoptosis.

Statistical analysis. Data are presented as the mean \pm SD. Comparisons among multiple groups were conducted using one-way ANOVA followed by Tukey's post hoc test. $\mathrm{P}<0.05$ was considered to indicate a statistically significant difference. Statistical analyses were performed using SPSS software (version 17.0; SPSS, Inc.). All experiments were performed in triplicate.

\section{Results}

Lyc mitigates HG-induced MPC5 podocyte injury. Cell viability was not significantly different between the NG and HP groups, which indicated that HP had a limited effect on MPC5 podocyte viability (Fig. 1A; P>0.05). However, cell viability in the HG group was significantly reduced compared with the NG group (Fig. 1A; $\mathrm{P}<0.01$ ). Compared with the HG group, cell viability in the HG + L-Lyc group was not significantly increased (Fig. 1A; $>>0.05$ ), whereas cell viability was significantly increased in the HG + H-Lyc group (Fig. $1 \mathrm{~A} ; \mathrm{P}<0.01$ ), which indicated that Lyc may increase MPC5 podocyte viability under HG conditions.

Compared with the NG group, the relative protein expression levels of nephrin and podocin were significantly reduced in the HG group (Fig. $1 \mathrm{~B}$ and $\mathrm{C} ; \mathrm{P}<0.05$ and $\mathrm{P}<0.01$, respectively); however, L-Lyc or H-Lyc significantly reversed HG-mediated effects on nephrin and podocin expression (Fig. 1B and $\mathrm{C} ; \mathrm{P}<0.05$ and $\mathrm{P}<0.01$, respectively).

Lyc attenuates HG-induced MPC5 podocyte apoptosis. The effect of HG and Lyc on MPC5 podocyte apoptosis was assessed by flow cytometry (Fig. 2A and B). The HG group displayed a significantly increased rate of apoptosis compared with the NG group $(\mathrm{P}<0.01)$, which was significantly reduced by the addition of L-Lyc or H-Lyc (Fig. 2A and B; P<0.05 and $\mathrm{P}<0.01$, respectively). Subsequently, the expression levels of apoptosis-related proteins (Bcl-2, Bax and C caspase-3) in the different groups were measured. Compared with the NG group, the protein expression levels of $\mathrm{Bax}$ and $\mathrm{C}$ caspase- 3 were significantly increased (Fig. $2 \mathrm{C}$ and $\mathrm{D} ; \mathrm{P}<0.01$ ), whereas the protein expression levels of $\mathrm{Bcl}-2$ were significantly decreased in the $\mathrm{HG}$ group (Fig. $2 \mathrm{C}$ and $\mathrm{D} ; \mathrm{P}<0.01$ ). However, the presence of L-Lyc or H-Lyc significantly reversed HG-mediated effects on the expression of apoptosis-related proteins (Fig. 2C and D; all $\mathrm{P}<0.05)$.

Lyc attenuates $H G$-induced MPC5 podocyte apoptosis via activation of the PI3K/AKT signaling pathway. In order to investigate the mechanism underlying Lyc-mediated inhibition 

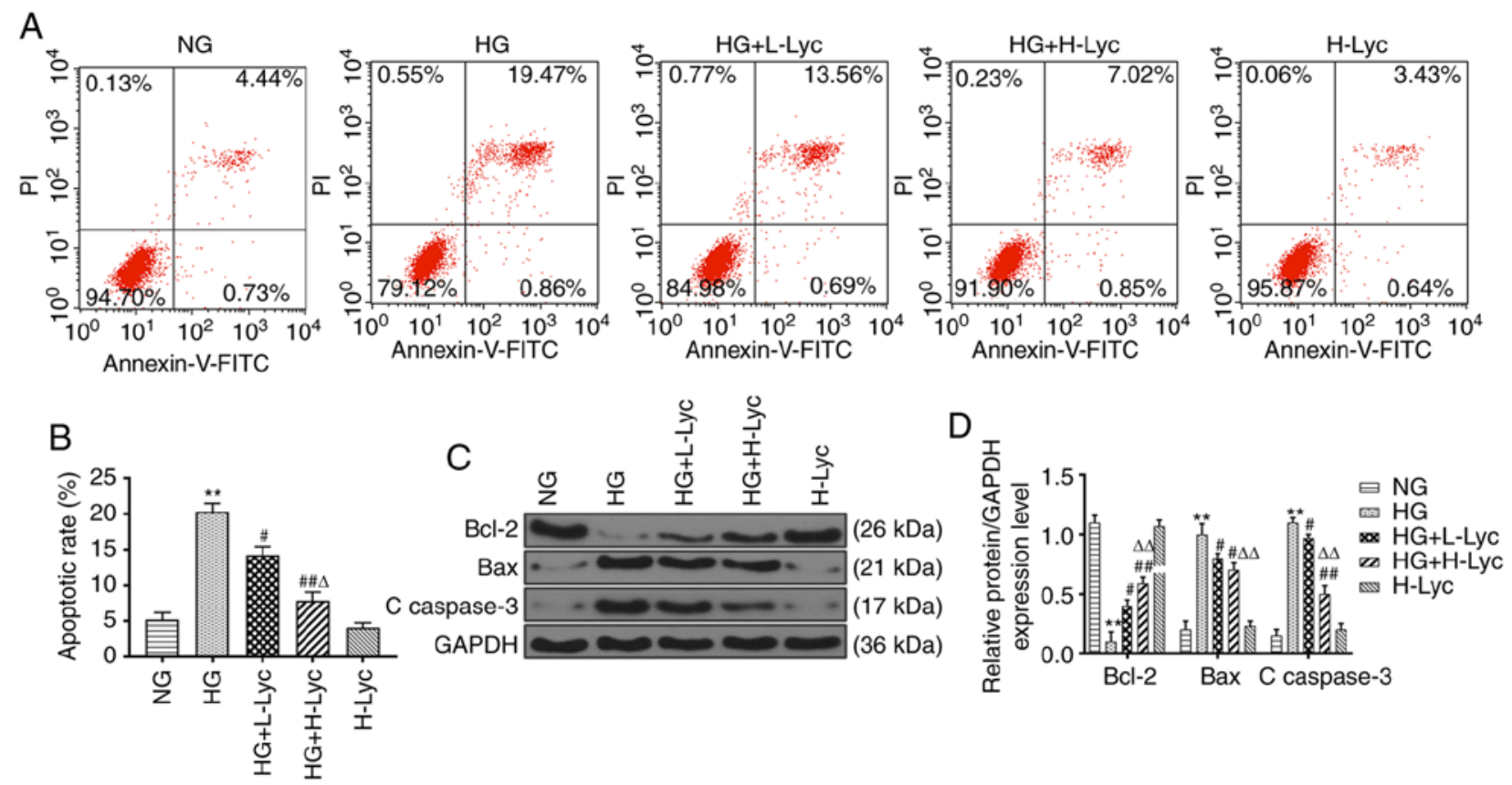

Figure 2. Lyc protects against HG-induced MPC5 podocyte apoptosis. MPC5 podocytes were divided into the following groups: i) NG (5.5 mM glucose); ii) HG (25 mM glucose); iii) HG + L-Lyc (25 mM glucose + 3.125 mM Lyc); iv) HG + H-Lyc (25 mM glucose + $12.5 \mathrm{mM} \mathrm{Lyc);} \mathrm{and} \mathrm{v)} \mathrm{H-Lyc} \mathrm{(12.5} \mathrm{mM} \mathrm{Lyc).}$ The rate of MPC5 podocyte apoptosis was (A) determined by flow cytometry and (B) quantified. The protein expression levels of antiapoptotic (Bcl-2) and proapoptotic (Bax and C caspase-3) proteins were (C) determined by western blotting and (D) semi-quantified. ${ }^{* *} \mathrm{P}<0.01 \mathrm{vs}$. NG; ${ }^{\#} \mathrm{P}<0.05$ and ${ }^{\# \#} \mathrm{P}<0.01 \mathrm{vs}$. $\mathrm{HG}$; ${ }^{\Delta} \mathrm{P}<0.05$ and ${ }^{\Delta \Delta} \mathrm{P}<0.01$ vs. H-Lyc. Lyc, lycopene; NG, normal glucose; HG, high glucose; L-Lyc, low-concentration lycopene; H-Lyc, high-concentration lycopene; $\mathrm{C}$ caspase-3, cleaved caspase-3; PI, propidium iodide.

of HG-induced MPC5 podocyte apoptosis, the expression levels of PI3K/AKT signaling pathway-related proteins were measured via western blotting. Compared with the NG group, the protein expression levels of p-PI3K and p-AKT were significantly downregulated in the HG group (Fig. 3A-D; $\mathrm{P}<0.01$ ). By contrast, compared with the HG group, the protein expression levels of p-PI3K and p-AKT were significantly upregulated in the HG + H-Lyc group (Fig. 3A-D; $\mathrm{P}<0.01$ ), but significantly downregulated in the $\mathrm{HG}+\mathrm{LY}$ group (Fig. 3A-D; $\mathrm{P}<0.05$ and $\mathrm{P}<0.01$, respectively). Moreover, compared with the HG + H-Lyc group, the expression levels of p-PI3K and p-AKT were significantly downregulated in the HG + H-Lyc + LY group (Fig. 3A-D; P<0.01). In addition, HG-mediated downregulation of p-PI3K and p-AKT expression levels was enhanced by LY, with the HG + LY group displaying significantly decreased p-PI $3 \mathrm{~K}$ and $\mathrm{p}-\mathrm{AKT}$ expression levels compared with the HG group (Fig. 3A-D; $\mathrm{P}<0.05$ and $\mathrm{P}<0.01$, respectively). However, under HG conditions, Lyc increased the expression levels of p-PI3K and p-AKT in MPC5 podocytes but LY inhibited Lyc-mediated upregulation (Fig. 3A-D).

Flow cytometry analysis indicated that the HG group displayed significantly increased rates of MPC5 podocyte apoptosis compared with those in the NG group, whereas the $\mathrm{HG}+\mathrm{H}-\mathrm{Lyc}+\mathrm{LY}$ group displayed a significantly reduced rate of MPC5 podocyte apoptosis compared with that in the HG + LY group (Fig. 3E and F; P<0.01). HG + H-Lyc + LY group exhibited significantly increased rates of MPC5 podocyte apoptosis compared with those in the HG + H-Lyc group (Fig. 3E and $\mathrm{F} ; \mathrm{P}<0.05$ ). In addition the expression levels of nephrin and podocin were significantly reduced in the HG group compared with the NG group. Compared with those in the HG group, the expression levels of nephrin and podocin were significantly increased in the HG $+\mathrm{H}$-Lyc group (Fig. 3G and $\mathrm{H} ; \mathrm{P}<0.01$ ), but were significantly reduced in the $\mathrm{HG}+\mathrm{LY}$ group compared with HG group (Fig. 3G and H; $\mathrm{P}<0.05$, respectively). However, the HG $+\mathrm{H}-\mathrm{Lyc}+\mathrm{LY}$ group displayed significantly increased nephrin and podocin expression levels compared with the HG + LY group (Fig. 3G and H; $\mathrm{P}<0.01)$.

Lyc mitigates $H G$-induced MPC5 podocyte apoptosis by promoting autophagy. The expression levels of autophagy-related proteins were measured via western blotting. The protein expression levels of LC3II/LC3I and Beclin-1 were significantly upregulated in the $\mathrm{HG}$ group compared with the NG group (Fig. 4A-C; $\mathrm{P}<0.05$ and $\mathrm{P}<0.01$, respectively). Compared with the HG group, the protein expression levels of LC3II/LC3I and Beclin-1 were significantly increased in the HG + H-Lyc group $(\mathrm{P}<0.01)$, but significantly decreased in the HG + LY group $(\mathrm{P}<0.05)$. Moreover, the effects of LY on Beclin-1 and LC3II/LC3I expression levels were significantly inhibited in the presence of Lyc under HG conditions (Fig. 4A-C; $\mathrm{P}<0.05$ and $\mathrm{P}<0.01$, respectively).

Autophagy is associated with cell apoptosis (22), so whether 3-methyladenine (3-MA), an autophagy inhibitor, altered the protective effect of Lyc on HG-induced MPC5 podocyte apoptosis was investigated. The $\mathrm{HG}+3-\mathrm{MA}$ group displayed significantly increased rates of MPC5 podocyte apoptosis compared with the HG group (Fig. 4D and E; $\mathrm{P}<0.05)$. Pretreament with $3-\mathrm{MA}$ inhibited the protective effect of Lyc on HG-induced MPC5 podocyte apoptosis 


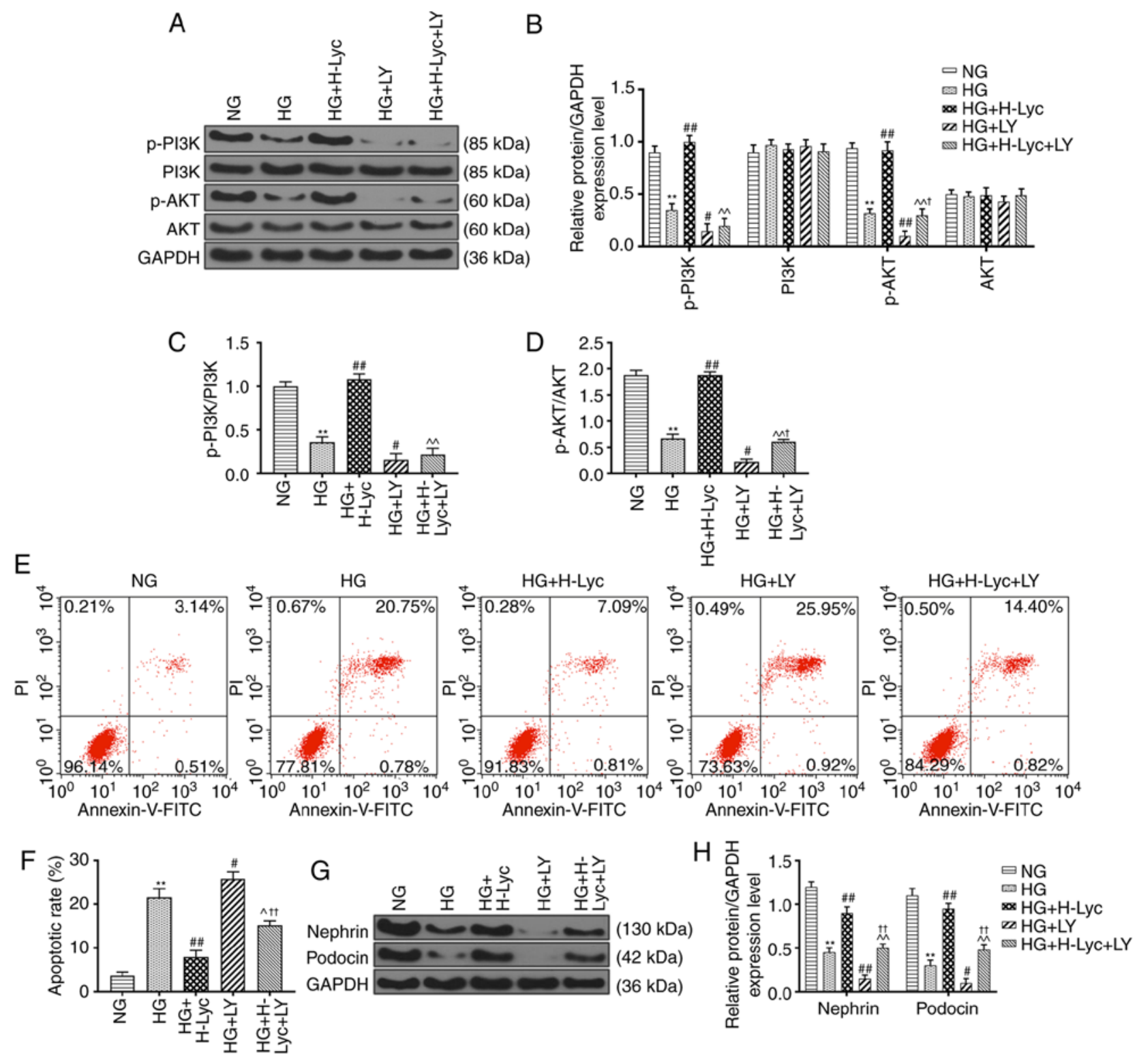

Figure 3. Lyc enhances PI3K/AKT activation and inhibits HG-induced MPC5 podocyte apoptosis. MPC5 podocytes were divided into the following groups: i) NG (5.5 mM glucose); ii) HG (25 mM glucose); iii) HG + H-Lyc ( $25 \mathrm{mM}$ glucose $+12.5 \mathrm{mM} \mathrm{Lyc})$; iv) HG + LY ( $25 \mathrm{mM}$ glucose $+20 \mu \mathrm{M} \mathrm{LY})$; and v) HG + H-Lyc + LY (25 mM glucose + $12.5 \mathrm{mM}$ Lyc $+20 \mu \mathrm{M} \mathrm{LY})$. Protein expression levels of p-PI3K, PI3K, p-AKT and AKT were (A) determined by western blotting and (B) quantified. The ratios of (C) p-PI3K/PI3K and (D) p-AKT/AKT. The rate of apoptosis was (E) determined by flow cytometry and (F) quantified. Protein expression levels of nephrin and podocin were $(\mathrm{G})$ determined by western blotting and $(\mathrm{H})$ quantified. ${ }^{* *} \mathrm{P}<0.01 \mathrm{vs}$. NG; ${ }^{\# P} \mathrm{P}<0.05$ and ${ }^{\# \#} \mathrm{P}<0.01$ vs. HG; ${ }^{\wedge} \mathrm{P}<0.05$ and ${ }^{\wedge} \mathrm{P}<0.01$ vs. HG $+\mathrm{H}-\mathrm{Lyc} ;{ }^{\dagger} \mathrm{P}<0.05$ and ${ }^{\dagger} \mathrm{P}<0.01$ vs. HG + LY. Lyc, lycopene; NG, normal glucose; HG, high glucose; H-Lyc, high-concentration lycopene; LY, LY294002; p, phosphorylated; PI, propidium iodide.

(Fig. 4D and E; $\mathrm{P}<0.01$ ). In addition, compared with the HG group, the protein expression levels of LC3II/LC3I and Beclin-1 were significantly increased in the HG + H-Lyc group, but significantly reduced in the $\mathrm{HG}+3-\mathrm{MA}$ group (Fig. 5A-C; $\mathrm{P}<0.01$ ). Moreover, 3-MA partially reversed H-Lyc-mediated effects on autophagy-related protein expression under HG conditions (Fig. 5A-C; $\mathrm{P}<0.01$ ). Furthermore, compared with the HG group, the $\mathrm{HG}+\mathrm{H}$-Lyc group displayed significantly increased podocin and nephrin expression levels $(\mathrm{P}<0.01)$, whereas the $\mathrm{HG}+3$-MA group displayed significantly decreased expression levels of podocin and nephrin (Fig. 5D-E; P<0.01). In addition, 3-MA weakened $\mathrm{H}$-Lyc-induced podocin and nephrin expression (Fig. 5D-E; $\mathrm{P}<0.01$ ).
Therefore, the results suggested that HG and Lyc significantly enhanced MPC5 podocyte autophagy, which may be crucial for the protective effects of Lyc against HG-induced MPC5 podocyte apoptosis.

\section{Discussion}

The function of a podocyte depends on the cytoskeleton and certain marker proteins of podocytes, such as nephrin and podocin (23). Nephrin and podocin proteins function as glomerular filtration barriers, and their expression affects the permeability of the glomerular matrix membrane, which in turn affects kidney function $(24,25)$. Abnormal expression of nephrin and podocin is a marker of podocyte injury (26). 

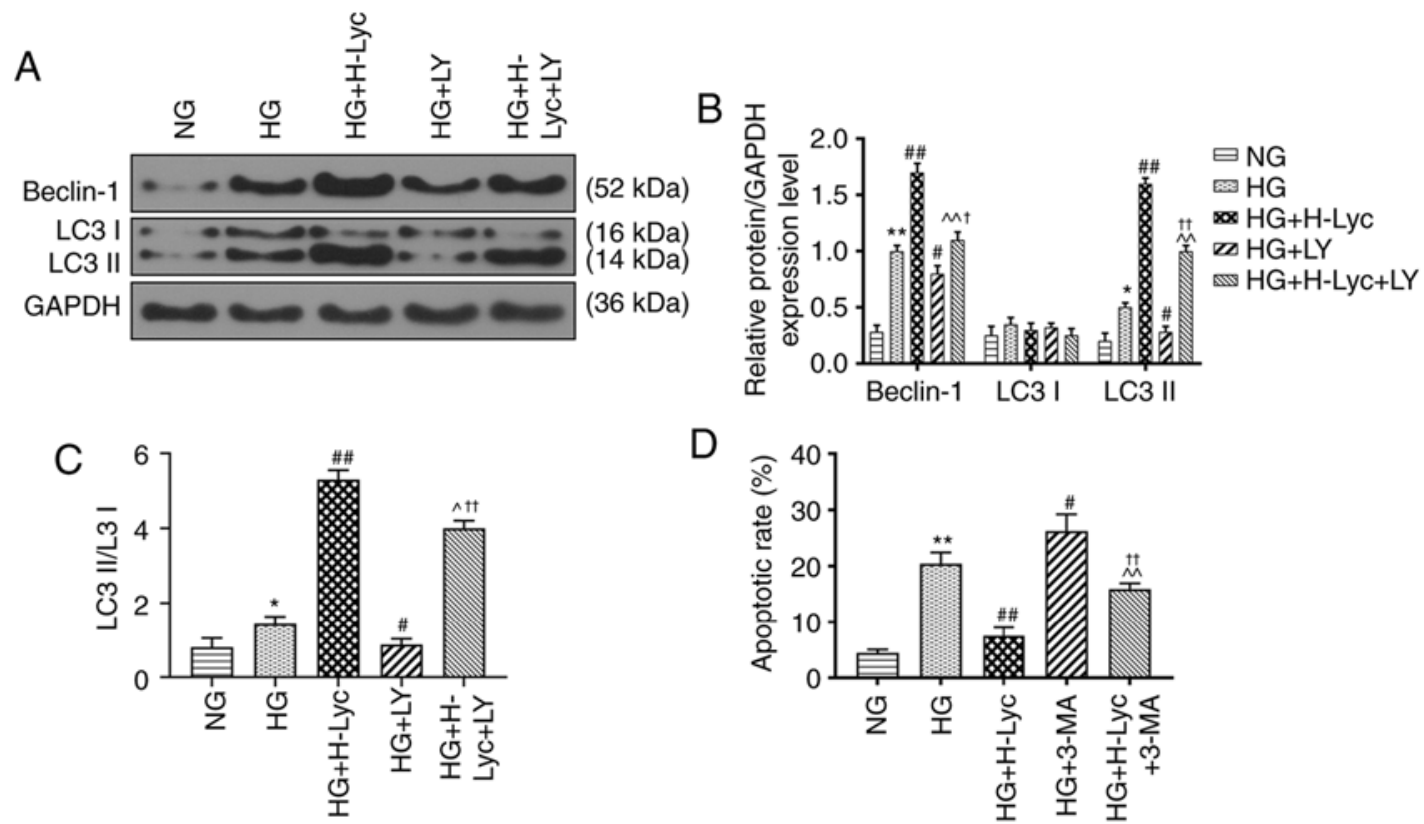

$\mathrm{D}$
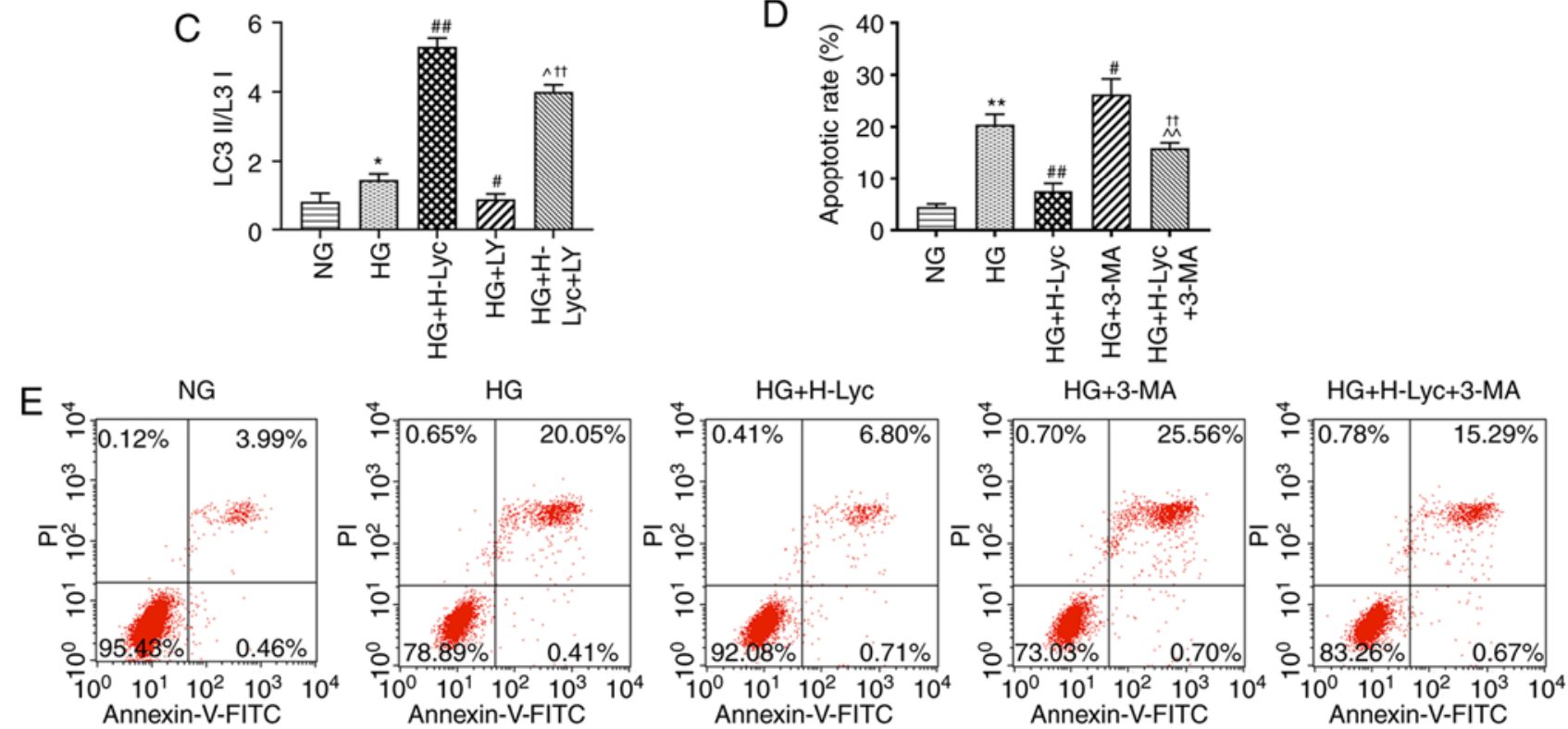

Figure 4. Lyc inhibits HG-induced MPC5 podocyte apoptosis by promoting autophagy via the PI3K/AKT signaling pathway. MPC5 podocytes were divided into the following groups: i) $\mathrm{NG}(5.5 \mathrm{mM}$ glucose); ii) HG (25 mM glucose); iii) HG + H-Lyc (25 mM glucose + $12.5 \mathrm{mM}$ Lyc); iv) HG + LY (25 mM glucose + $20 \mu \mathrm{M} \mathrm{LY})$; and v) HG + H-Lyc + LY (25 mM glucose + $12.5 \mathrm{mM} \mathrm{Lyc}+20 \mu \mathrm{M} \mathrm{LY})$. Protein expression levels of Beclin-1, LC3I and LC3II were (A) determined by western blotting and (B) quantified. (C) The ratio of LC3II/LC3I. ${ }^{*} \mathrm{P}<0.05$ and ${ }^{* * *} \mathrm{P}<0.01$ vs. NG; ${ }^{\#} \mathrm{P}<0.05$ and ${ }^{\# \#} \mathrm{P}<0.01$ vs. HG; ${ }^{\wedge} \mathrm{P}<0.05$ and ${ }^{\wedge \wedge} \mathrm{P}<0.01$ vs. HG + H-Lyc; ${ }^{\mathrm{P}} \mathrm{P}<0.05$ and ${ }^{\dagger} \mathrm{P}<0.01$ vs. HG + LY. MPC5 podocytes were divided into the following groups: i) NG (5.5 mM glucose); ii) HG (25 mM glucose); iii) HG + H-Lyc (25 mM glucose + $12.5 \mathrm{mM} \mathrm{Lyc})$; iv) HG + 3-MA (25 mM glucose + $5 \mathrm{mM} 3-\mathrm{MA})$; and v) HG + H-Lyc + 3-MA (25 mM glucose + $12.5 \mathrm{mM}$ Lyc +5 mM 3-MA). (D) The rate of apoptosis was determined by flow cytometry. (E) Representative scatter plots. ${ }^{* *} \mathrm{P}<0.01$ vs. NG; ${ }^{\# P}<0.05$ and ${ }^{\# \#} \mathrm{P}<0.01$ vs. HG; ${ }^{\wedge} \mathrm{P}<0.01$ vs. HG + H-Lyc; ${ }^{\circ} \mathrm{P}<0.01$ vs. HG + 3-MA. Lyc, lycopene; NG, normal glucose; HG, high glucose; H-Lyc, high-concentration lycopene; LY, LY294002; LC3, microtubule associated protein 1 light chain 3; 3-MA, 3-methyladenine; PI, propidium iodide.

Consistent with previous study (21), the present study demonstrated that Lyc significantly reversed the inhibitory effects of HG on cell viability, and nephrin and podocin expression in podocytes.

Podocyte loss is one of the most important pathological alterations that occurs in DN (27). As a key event during DN progression, HG-induced apoptosis is often accompanied by podocyte loss, which is an early feature of DN and is highly predictive of the disease progression (28-30). Apoptosis-defined programmed cell death is also caused by external killers in certain circumstances (31). For example, irradiation or drugs used for cancer chemotherapy results in DNA damage in some cells, which can lead to apoptotic death (32). In the present study, the results indicated that HG promoted MPC5 podocyte apoptosis, and Lyc mitigated MPC5 podocyte apoptosis under HG conditions. Apoptosis can be regulated via the PI3K/AKT signaling pathways (33) and PI3K/AKT can be activated by a number of different cellular stimuli or toxic insults $(34,35)$. Previous studies have reported that Lyc can regulate PI3K/AKT signaling pathways in prostate cancer and human mesenchymal stem cells $(36,37)$. To further explore the mechanism underlying the effects of Lyc on HG-induced MPC5 podocyte apoptosis, a PI3K inhibitor was used. Lyc activated the PI3K/AKT signaling pathway, which inhibited HG-mediated MPC5 podocyte apoptosis. Similarly, a previous study reported that Lyc reduced tert-Butyl hydroperoxide-induced cell apoptosis, which is possibly related to activation of the PI3K/AKT signaling pathway (38). However, Chan et al (39) reported that Lyc can inhibit the PI3K/AKT signaling pathway during platelet-derived growth factor-BB-induced retinal pigment epithelial cell migration. In addition, the results of the present study suggested that Lyc treatment had no significant effect on cell viability or the expression of nephrin and podocin in MPC5 podocytes compared with the NG and HG groups; however, under HG conditions, Lyc treatment promoted MPC5 podocyte cell viability, and nephrin and podocin protein expression. Li et al (40) reported that Lyc treatment had no significant effect on ameloblast apoptosis, whereas Lyc attenuated fluoride-induced ameloblasts apoptosis. Collectively, the 


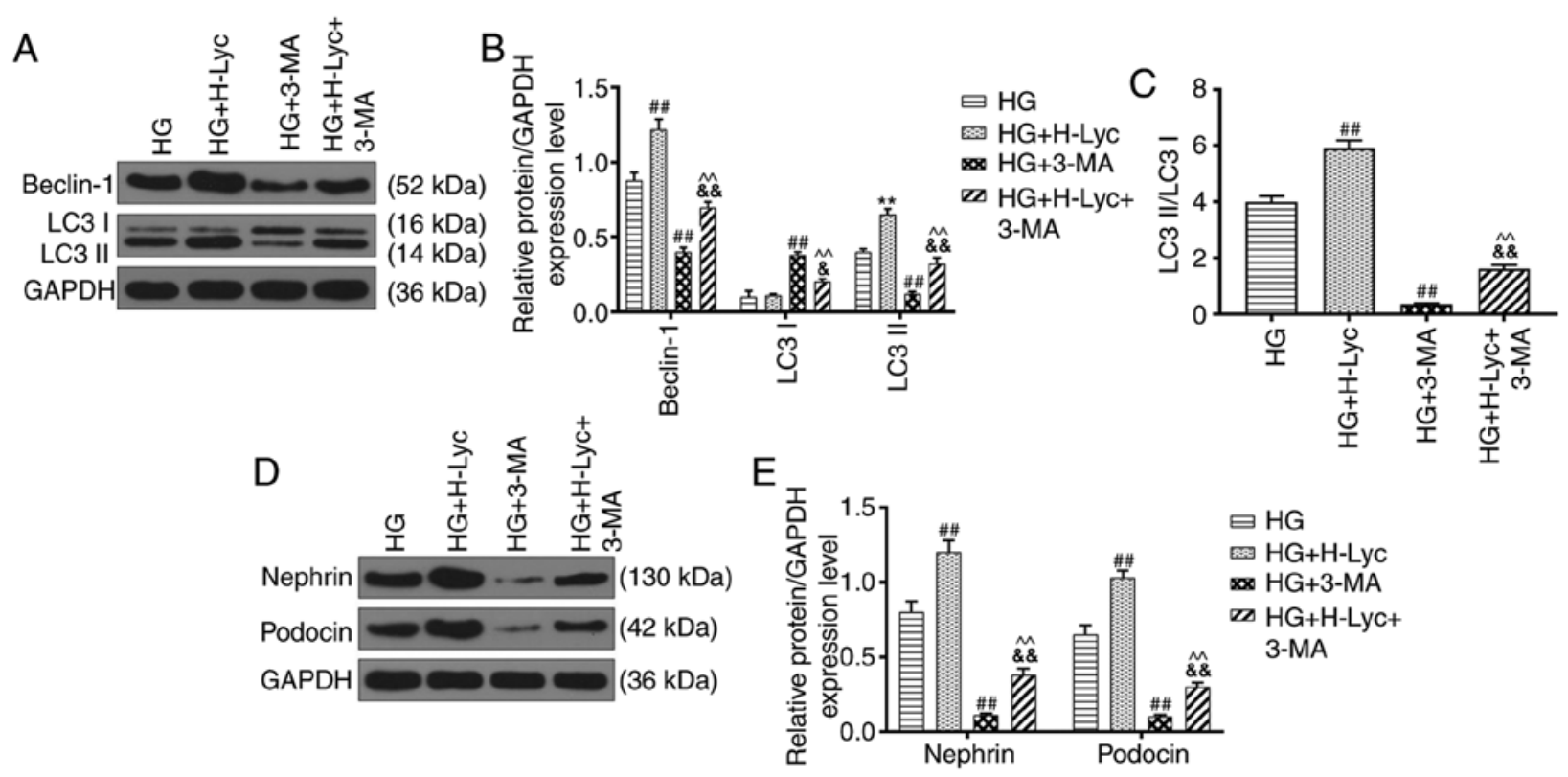

Figure 5. Lyc promotes the expression of nephrin, podocin and autophagy-related proteins in MPC5 podocytes. MPC5 podocytes were divided into the following groups: i) HG (25 mM glucose); ii) $\mathrm{HG}+\mathrm{H}-\mathrm{Lyc}(25 \mathrm{mM}$ glucose + $12.5 \mathrm{mM} \mathrm{Lyc})$; iii) $\mathrm{HG}+3-\mathrm{MA}(25 \mathrm{mM}$ glucose + $5 \mathrm{mM} \mathrm{3-MA})$; and HG + H-Lyc + 3-MA (25 mM glucose + $12.5 \mathrm{mM} \mathrm{Lyc}+5 \mathrm{mM}$ 3-MA). Protein expression levels of Beclin-1, LC3II and LC3I were (A) determined by western blotting and (B) semi-quantified. (C) The ratio of LC3BII/LC3BI. Protein expression levels of nephrin and podocin were (D) determined by western blotting and (E) semi-quantified. ${ }^{\# \#} \mathrm{P}<0.01$ vs. HG; ${ }^{\wedge} \mathrm{P}<0.01$ vs. HG $+\mathrm{H}-\mathrm{Lyc} ;{ }^{\circledR} \mathrm{P}<0.05$ and ${ }^{\& \&} \mathrm{P}<0.01$ vs. HG + 3-MA. Lyc, lycopene; HG, high glucose; H-Lyc, high-concentration lycopene; 3-MA, 3-methyladenine; LC3, microtubule associated protein 1 light chain 3.

aforementioned findings indicated that Lyc may serve a role under certain pathological conditions.

As the inhibition of autophagy is highly associated with triggering cell apoptosis, and both apoptosis and autophagy are involved in cell growth, differentiation, and death (41), whether autophagy regulated the protective effect of Lyc on HG-induced MPC5 podocyte apoptosis was investigated. Autophagy is a natural process related to numerous human diseases, which can protect the human body from cell injury and death (42). Therefore, autophagic activity of podocytes serves a protective role in renal injury and could delay the progression of podocytopathies (43). Cell apoptosis and autophagy are complex regulatory processes that involve a number of upstream regulatory signaling pathways, including the PI3K/AKT signaling pathway (44). A previous study demonstrated that enhancing autophagy protects against palmitic acid-mediated podocyte apoptosis (45), and progranulin alleviates HG-induced human podocyte injury by regulating CAMKK/AMPK-mediated autophagy (46). Therefore, promoting autophagy could attenuate HG-induced podocyte apoptosis (47). Moreover, resveratrol protected against podocyte apoptosis by activating autophagy in DN model mice (48). Consistently, another study reported that Lyc protected against apoptosis by increasing autophagy in hypoxia/reoxygenation-induced H9C2 myocardioblast cells (49). However, Kobayashi et al (50) reported that suppression of autophagy exerted protective effects against HG-mediated cardiomyocyte injury. In the present study, the results also indicated that the HG group displayed increased apoptosis compared with the NG group, and promotion of autophagy promotes apoptosis, which is a stress phenomenon (51).

In the present study, 3-MA, an autophagy inhibitor, was used to explore the mechanism underlying the effect of Lyc on autophagy. The results indicated that promoting autophagy by Lyc attenuated HG-mediated MPC5 podocyte apoptosis, which was associated with activation of the PI3K/AKT signaling pathway. It has been reported that Lyc relieved HG-mediated podocyte injury by promoting MPC5 podocyte autophagy, which may involve the PI3K/AKT signaling pathway (21). Similarly, a previous study reported that notoginsenoside R1 attenuated glucose-mediated podocyte injury by reducing apoptosis and promoting autophagy via activation of the PI3K/AKT/mTOR signaling pathway (11). However, in colorectal cancer cells, autophagy and apoptosis were promoted by Grape seed procyanidin B2 via regulation of the PI3K/AKT signaling pathway (44).

In conclusion, the present study indicated that Lyc protected against HG-induced MPC5 podocyte injury and attenuated HG-induced MPC5 podocyte apoptosis by promoting autophagy via activation of the PI3K/AKT signaling pathway. The present study demonstrated a potential mechanism underlying the therapeutic effect of Lyc on DN, which may serve as a potential therapeutic target for DN progression.

\section{Acknowledgements}

Not applicable.

\section{Funding}

No funding was received.

\section{Availability of data and materials}

The datasets used and/or analyzed during the current study are available from the corresponding author on reasonable request. 


\section{Authors' contributions}

QW substantially contributed to the conception and design of the study. RL, ZX and $\mathrm{CH}$ acquired, analyzed and interpreted the data. QW drafted the manuscript and critically revised it for important intellectual content. All authors read and approved the final manuscript.

\section{Ethics approval and consent to participate}

Not applicable.

\section{Patient consent for publication}

Not applicable.

\section{Competing interests}

The authors declare that they have no competing interests.

\section{References}

1. Jin Y, Liu S, Ma Q, Xiao D and Chen L: Berberine enhances the AMPK activation and autophagy and mitigates high glucose-induced apoptosis of mouse podocytes. Eur J Pharmacol 794: 106-114, 2017.

2. Hazman Ö and Bozkurt MF: Anti-inflammatory and antioxidative activities of safranal in the reduction of renal dysfunction and damage that occur in diabetic nephropathy. Inflammation 38: $1537-1545,2015$.

3. Aso Y: Cardiovascular disease in patients with diabetic nephropathy. Curr Mol Med 8: 533-543, 2008

4. Lee JH, Kim D, Oh YS and Jun HS: Lysophosphatidic acid signaling in diabetic nephropathy. Int J Mol Sci 20: 2850, 2019.

5. Vervoort G: Treatment Goals in Diabetic Nephropathy. Pathophysiology and Clinical Aspects. In: Diabetic Nephropathy. Roelofs J and Vogt L (eds). Springer, Cham, pp435-450, 2019.

6. Najafian B, Alpers CE and Fogo AB: Pathology of human diabetic nephropathy. Contrib Nephrol 170: 36-47, 2011.

7. Ziyadeh FN, Hoffman BB, Han DC, Iglesias-De La Cruz MC, Hong SW, Isono $\mathrm{M}$, Chen S, McGowan TA and Sharma K: Long-term prevention of renal insufficiency, excess matrix gene expression, and glomerular mesangial matrix expansion by treatment with monoclonal antitransforming growth factor-beta antibody in $\mathrm{db} / \mathrm{db}$ diabetic mice. Proc Natl Acad Sci USA 97: 8015-8020, 2000.

8. Yu SM and Bonventre JV: Acute kidney injury and progression of diabetic kidney disease. Adv Chronic Kidney Dis 25: 166-180, 2018.

9. Tharaux PL and Huber TB: How is proteinuric diabetic nephropathy caused by disturbed proteostasis and autophagy in podocytes? Diabetes 65: 539-541, 2016.

10. Marusugi K, Nakano K, Sasaki H,Kimura J, Yanobu-Takanashi R, Okamura T and Sasaki N: Functional validation of tensin2 SH2-PTB domain by CRISPR/Cas9-mediated genome editing. J Vet Med Sci 78: 1413-1420, 2016.

11. Huang G, Zou B, Lv J, Li T, Huai G, Xiang S, Lu S, Luo H, Zhang Y, Jin Y and Wang Y: Notoginsenoside R1 attenuates glucose-induced podocyte injury via the inhibition of apoptosis and the activation of autophagy through the PI3K/Akt/mTOR signaling pathway. Int J Mol Med 39: 559-568, 2017.

12. Barisoni L, Schnaper HW and Kopp JB: Advances in the biology and genetics of the podocytopathies: Implications for diagnosis and therapy. Arch Pathol Lab Med 133: 201-216, 2009.

13. Shankland SJ: The podocyte's response to injury: Role in proteinuria and glomerulosclerosis. Kidney Int 69: 2131-2147, 2006.

14. Agarwal S and Rao AV: Tomato lycopene and its role in human health and chronic diseases. CMAJ 163: 739-744, 2000.

15. Fan S, Sun JB, Li R, Song X and Li J: Lycopene protects myocardial ischemia injury through anti-apoptosis and anti-oxidative stress. Eur Rev Med Pharmacol Sci 23: 3096-3104, 2019.

16. Sahin K, Orhan C, Sahin N and Kucuk O: Anticancer Properties of Lycopene. Springer, Cham, 2019.
17. Kawata A, Murakami Y, Suzuki $S$ and Fujisawa $S$ : Anti-inflammatory activity of $\beta$-Carotene, Lycopene and Tri-n-butylborane, a scavenger of reactive oxygen species. In Vivo 32: 255-264, 2018.

18. Li W, Wang G, Lu X, Jiang Y, Xu L and Zhao X: Lycopene ameliorates renal function in rats with streptozotocin-induced diabetes. Int J Clin Exp Pathol 7: 5008-5015, 2014.

19. Ni L, Yang XL, Qiao H and Lin J: Effects of lycopene on high glucose-induced autophagy and apoptosis of podocytes. Clin Nephrol 18: 48-53, 2018 (In Chinese).

20. Liu WT, Peng FF, Li HY, Chen XW, Gong WQ, Chen WJ, Chen YH, Li PL, Li ST, Xu ZZ and Long HB: Metadherin facilitates podocyte apoptosis in diabetic nephropathy. Cell Death Dis 7: e2477, 2016

21. Ni L, Qiao H, Lin J and Chang-xuan L: Lycopene relieves the damage of mouse podocytes induced by high glucose and its mechanism. Southeast Univ (Med Sci Edi) 37: 229-234, 2018 (In Chinese).

22. Wang K: Autophagy and apoptosis in liver injury. Cell Cycle 14: 1631-1642, 2015.

23. Gao F, He X, Liang S, Liu S, Liu H, He Q, Chen L, Jiang H and Zhang Y: Quercetin ameliorates podocyte injury via inhibition of oxidative stress and the TGF- $\beta 1 /$ Smad pathway in DN rats. RSC Adv 8: 35413-35421, 2018.

24. Wei M, Li Z, Xiao L and Yang Z: Effects of ROS-relative NF-KB signaling on high glucose-induced TLR4 and MCP-1 expression in podocyte injury. Mol Immunol 68: 261-271, 2015.

25. Gong Q, He LL, Wang ML, Ouyang H, Gao HW, Feng YL, Yang SL, Du LJ, Li J and Luo YY: Anemoside B4 protects rat kidney from adenine-induced injury by attenuating inflammation and fibrosis and enhancing podocin and nephrin expression. Evid Based Complement Alternat Med 2019: 8031039, 2019.

26. Tesch GH: Review: Serum and urine biomarkers of kidney disease: A pathophysiological perspective. Nephrology (Carlton) 15: 609-616, 2010.

27. Szrejder M and Piwkowska A: AMPK signalling: Implications for podocyte biology in diabetic nephropathy. Biol Cell 111: 109-120, 2019.

28. Reidy K, Kang HM, Hostetter T and Susztak K: Molecular mechanisms of diabetic kidney disease. J Clin Invest 124: 2333-2340, 2014.

29. Li W, Wang Q, Du M, Ma X, Wu L, Guo F, Zhao S, Huang F, Wang $\mathrm{H}$ and Qin G: Effects of overexpressing FoxO1 on apoptosis in glomeruli of diabetic mice and in podocytes cultured in high glucose medium. Biochem Biophys Res Commun 478: 612-617, 2016.

30. Saurus P, Kuusela S, Dumont V, Lehtonen E, Fogarty CL, Lassenius MI, Forsblom C, Lehto M, Saleem MA, Groop PH and Lehtonen S: Cyclin-dependent kinase 2 protects podocytes from apoptosis. Sci Rep 6: 21664, 2016.

31. Nagata S: Apoptosis by death factor. Cell 88: 355-365, 1997.

32. Fulda S: Therapeutic exploitation of necroptosis for cancer therapy. Semin Cell Dev Biol 35: 51-56, 2014

33. Dey JH, Bianchi F, Voshol J, Bonenfant D, Oakeley EJ and Hynes NE: Targeting fibroblast growth factor receptors blocks PI3K/AKT signaling, induces apoptosis, and impairs mammary tumor outgrowth and metastasis. Cancer Res 70: 4151-4162, 2016.

34. Song H, Fu Y, Wan D, Xia W, Lyu F, Liu L and Shen L: Mytoxin B and myrothecine $\mathrm{A}$ induce apoptosis in human hepatocarcinoma cell line SMMC-7721 via PI3K/Akt signaling pathway. Molecules 24: 2291, 2019.

35. Porta C and Figlin RA: Phosphatidylinositol-3-Kinase/Akt signaling pathway and kidney cancer, and the therapeutic potential of phosphatidylinositol-3-Kinase/Akt inhibitors. J Urol 182: 2569-2577, 2009.

36. Chen J, O'Donoghue A, Deng YF, Zhang B, Kent F and O'Hare T: The effect of lycopene on the PI3K/Akt signalling pathway in prostate cancer. Anticancer Agents Med Chem 14: 800-805, 2014.

37. Ji YK, Lee JS, Han YS, Lee JH, Bae I, Yoon YM, Kwon SM and Lee SH: Pretreatment with lycopene attenuates oxidative Stress-Induced apoptosis in human mesenchymal stem cells. Biomol Ther (Seoul) 23: 517-524, 2015.

38. Huang C, Wen C, Yang M, Gan D, Fan C, Li A, Li Q, Zhao J, Zhu L and Lu D: Lycopene protects against t-BHP-induced neuronal oxidative damage and apoptosis via activation of the PI3K/Akt pathway. Mol Biol Rep 46: 3387-3397, 2019.

39. Chan CM, Fang JY, Lin HH, Yang CY and Hung CF: Lycopene inhibits PDGF-BB-induced retinal pigment epithelial cell migration by suppression of PI3K/Akt and MAPK pathways. Biochem Biophys Res Commun 388: 172-176, 2009. 
40. Li W, Jiang B, Cao X, Xie Y and Huang T: Protective effect of lycopene on fluoride-induced ameloblasts apoptosis and dental fluorosis through oxidative stress-mediated Caspase pathways. Chem Biol Interact 261: 27-34, 2017.

41. Fiandalo MV and Kyprianou N: Caspase control: Protagonists of cancer cell apoptosis. Exp Oncol 34: 165-175, 2012.

42. Murrow L and Debnath J: Autophagy as a stress-response and quality-control mechanism: Implications for cell injury and human disease. Annu Rev Pathol 8: 105-137, 2013.

43. Zeng C, Fan Y, Wu J, Shi S, Chen Z, Zhong Y, Zhang C, Zen K and Liu Z: Podocyte autophagic activity plays a protective role in renal injury and delays the progression of podocytopathies. J Pathol 234: 203-213, 2014.

44. Zhang R, Yu Q, Lu W, Shen J, Zhou D, Wang Y, Gao S and Wang Z: Grape seed procyanidin B2 promotes the autophagy and apoptosis in colorectal cancer cells via regulating PI3K/Akt signaling pathway. Onco Targets Ther 12: 4109-4118, 2019.

45. Jiang XS, Chen XM, Wan JM, Gui HB, Ruan XZ and Du XG: Autophagy protects against palmitic acid-induced apoptosis in podocytes in vitro. Sci Rep 7: 42764, 2017.

46. Zhou D, Zhou M, Wang Z, Fu Y, Jia M, Wang X, Liu M, Zhang Y, Sun Y, Zhou Y, et al: Progranulin alleviates podocyte injury via regulating CAMKK/AMPK-mediated autophagy under diabetic conditions. J Mol Med (Berl) 97: 1507-1520, 2019.
47. Dong $\mathrm{C}$, Zheng $\mathrm{H}$, Huang $\mathrm{S}$, You $\mathrm{N}$, Xu J, Ye $\mathrm{X}$, Zhu Q, Feng Y, You Q, Miao H, et al: Heme oxygenase-1 enhances autophagy in podocytes as a protective mechanism against high glucose-induced apoptosis. Exp Cell Res 337: 146-159, 2015.

48. Huang SS, Ding DF, Chen S, Dong CL, Ye XL, Yuan YG, Feng YM, You N, Xu JR, Miao H, et al: Resveratrol protects podocytes against apoptosis via stimulation of autophagy in a mouse model of diabetic nephropathy. Sci Rep 7: 45692, 2017.

49. Chen F, Sun ZW, Ye LF, Fu GS, Mou Y and Hu SJ: Lycopene protects against apoptosis in hypoxia/reoxygenation-induced H9C2 myocardioblast cells through increased autophagy. Mol Med Rep 11: 1358-1365, 2015.

50. Kobayashi S, Xu X, Chen K and Liang Q: Suppression of autophagy is protective in high glucose-induced cardiomyocyte injury. Autophagy 8: 577-592, 2012.

51. Chiarelli R, Martino C, Agnello M, Bosco L and Roccheri MC: Autophagy as a defense strategy against stress: Focus on paracentrotus lividus sea urchin embryos exposed to cadmium. Cell Stress Chaperones 21: 19-27, 2016.

(i) (9) This work is licensed under a Creative Commons EY No No Attribution-NonCommercial-NoDerivatives 4.0 International (CC BY-NC-ND 4.0) License. 